\title{
Formen der Hautalterung
}

\section{J. C. Simon}

Different Forms of Skin Aging

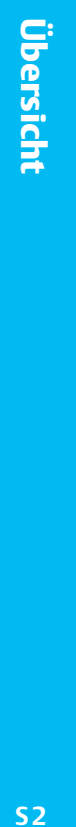

\section{Zusammenfassung}

Die Alterungsprozesse in der Haut spiegeln die auch in inneren Organen ablaufenden Vorgänge wider, sodass die Haut von Altersforschern bevorzugt als Modellsystem zur Klärung von Alterungsmechanismen herangezogen wird. Grundsätzlich unterscheidet man die intrinsische oder biologische Hautalterung von der extrinsischen, durch Umwelteinflüsse bedingten Alterung. Die vorliegende Arbeit stellt die verschiedenen Alterstheorien vor, die Altern entweder als genetisch bedingten Prozess oder als Abnutzungserscheinung erklären. Eine plausible Rolle wird heute den freien Radikalen zugeschrieben, die über die Induktion von DNA-Schäden zu einer nachhaltigen Beeinträchtigung von Körperfunktionen führen können.

\section{Abstract}

Human skin aging reflects processes going on in internal organs. Therefore skin is a favoured model elucidating mechanisms of aging. Basically the intrinsic or biological skin aging is distinguished from the extrinsic aging, caused by environmental factors. In this article different theories of aging are reviewed explaining aging as a genetic or abrasion process. A plausible role is attributed to free radicals. Free radicals are able to induce damage of DNA, which can lead to lasting adverse effects on bodily functions.

\section{Einleitung}

Dank der seit Beginn des 20. Jahrhunderts erreichten medizinischen Fortschritte hat unsere Lebenserwartung in den letzten Jahrzehnten immens zugenommen und ist weiterhin kontinuierlich im Anstieg. Im Jahr 2003 betrug der Anteil der über 60-Jährigen in Deutschland nach Angaben des Statistischen Bundesamtes bereits 17,5\%; er wird bis zum Jahr 2050 weiter auf rund ein Drittel wachsen [1]. Die frühere Tannenbaumform der Alterspyramide wird dann die so genannte Urnenform angenommen haben (Abb.1). Damit gewinnt das Thema „Altern“ für immer mehr Personen an Relevanz. Entsprechend erfreuen sich therapeutische wie kosmetische Maßnahmen, die ein gesundes Altern erlauben und den Körper möglichst lange jung, vital und attraktiv erhalten, ständig steigender Beliebtheit.
Im Rahmen des Alterungsprozesses kommt der Haut eine Sonderstellung zu, da sie als Außenbarriere des Organismus eine Schutzfunktion besitzt und damit im Gegensatz zu den meisten inneren Organen kontinuierlich und repetetiv einer Vielzahl externer Einflüsse ausgesetzt ist. Deshalb ist gerade die Haut für Altersforscher ein äußerst interessantes Forschungsobjekt: Sie kann als Spiegel für Alterungsprozesse des Körpers betrachtet werden, d.h. das Muster der Hautalterung erlaubt Rückschlüsse auf Alterungsmechanismen wie z.B. der inneren Organe.

\section{Prozesse der Hautalterung}

Im Rahmen des Alterungsprozesses ist zwischen intrinsischer und extrinsischer Hautalterung zu differenzieren. Im Verlauf der chronologischen intrinsischen Hautalterung („Zeitaltern“) läuft die durch genetische Prädisposition und Hormonstatus be- 


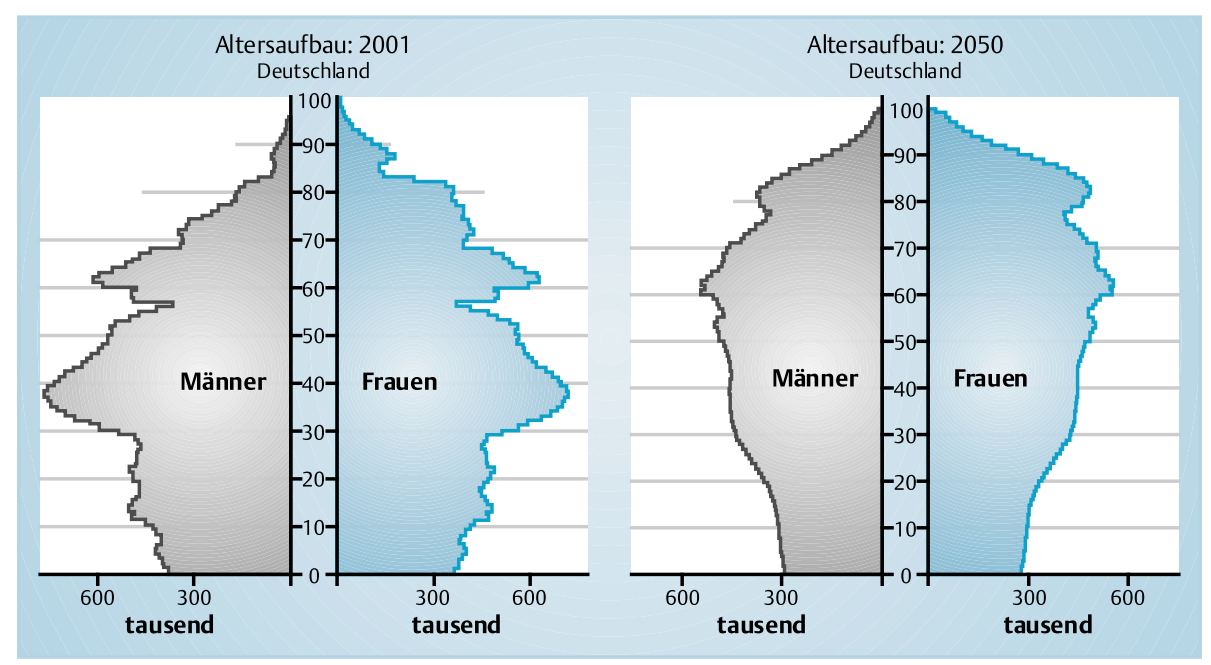

Abb. 1 Alterspyramide der Bundesrepublik Deutschland im Jahr 2001 und 2050: Der Anteil der über 60-jährigen Bundesbürger nimmt ständig zu [1].

stimmte biologische Uhr des menschlichen Organismus allmählich ab. Darüber hinaus werden Alterungsvorgänge der Haut zusätzlich durch externe Faktoren wie UV-Strahlung, Rauchen, Stress oder Alkohol ausgelöst. Dieses Altern durch Umwelteinflüsse wird als extrinsische Hautalterung („Umweltaltern“) bezeichnet und ist vielfach für die vorzeitige Alterung verantwortlich. Wichtigste Noxe in diesem Zusammenhang ist das UV-Licht (Photo-Aging), das zu Pigmentverschiebungen, trockener und glanzloser Haut und ausgeprägter Faltenbildung führt.

Im Unterschied dazu ist die intrinsische Hautalterung durch Schlaffheit, Trockenheit und Blässe mit Abnahme der Epidermisdicke um rund $50 \%$ und der Dermisdicke um rund $30 \%$ charakterisiert (Abb. 2). Da der stimulierende Effekt von Östrogenen bzw. Androgenen auf die Zellteilungsaktivität entfällt, nimmt die Zahl der Keratinozyten in der Basalzellschicht sowie der Melanozyten und Langerhans-Zellen in der Epidermis ab. Damit wird auch die Immunfunktion der Haut beeinträchtigt; die Wundheilung der Haut ist erschwert. Aufgrund der sinkenden Zahl und der nachlassenden Aktivität von Fibroblasten verringert sich der Kollagengehalt drastisch. Auch das subkutane Fettgewebe und das superfizielle Gefäßgeflecht verlieren an Stabilität. Diese Veränderungen resultieren in einer erhöhten Fragilität der Haut bereits bei Bagatelltraumen. Abnormale Nervenzellendigungen verleihen der Haut eine verstärkte Irritabilität. Auch die unter der Kontrolle von Sexualhormonen stehenden Talgdrüsen reduzieren ihre Funktion mehr und mehr. Gleichzeitig sinkt der Gehalt an Hyaluronsäure mit ihrer hohen Wasserbindungskapazität in der extrazellulären Matrix. Damit steigt die Trockenheit der Haut. Erschwerend hinzu kommen die Fragmentierung und zahlenmäßige Abnahme von Elastinfasern, was sich in einer verminderten Elastizität der Haut mit Schlaffheit und verstärkter Faltenbildung äußert [2].

Eine vergleichbare, allerdings etwas später einsetzende Entwicklung ist an der Urogenitalschleimhaut zu beobachten, die ebenfalls atrophiert und eine zunehmende Trockenheit zeigt. Parallel zur Abnahme des Hautkollagens sinkt auch die Knochendichte bei Frauen nach der Menopause drastisch ab [3]. Eine manifeste Osteoporose entwickelt sich jedoch erst 10 bis 15 Jahre nach Eintritt der Menopause; noch einige Jahre später manifestiert sich die Atherosklerose [4].
Um den durch die Veränderungen im Hormonhaushalt bedingten Alterungsprozessen in der Haut zu begegnen, wurde lange Zeit eine systemische Hormonersatztherapie zur Prävention und Behandlung von Folgen des Hormondefizits propagiert. In der Tat spielen derartige altersbedingte Veränderungen heute eine zunehmend wichtige Rolle, blieb doch das Menopausealter bei mitteleuropäischen Frauen in den letzten 100 Jahren mit durchschnittlich 51 Jahren praktisch konstant. Aufgrund der steigenden Lebenserwartung haben Frauen daher heute zum Zeitpunkt der Menopause noch über ein Drittel ihres Lebens vor sich [4], in denen sie mit den Konsequenzen der postmenopausalen Hormonveränderungen zurechtkommen müssen. Die in den letzten Jahren publizierten Ergebnisse großer randomisierter Studien zur Hormonersatztherapie, in denen die postulierten positiven Auswirkungen der systemischen Östrogensubstitution auf Herz-Kreislauf-System und Gehirn nicht verifiziert werden konnten, haben die Indikationen für diese Form der Prävention und Behandlung mittlerweile deutlich eingeschränkt. Zunehmendes Interesse gilt daher neuen alternativen Anti-Aging-Strategien. Derartigen Fragen ihrer Patienten müssen sich heute auch die Dermatologen stellen.

\section{Alterstheorien}

Jede Art von Lebewesen ist durch eine charakteristische maximale Lebensdauer gekennzeichnet. Diese maximal mögliche Lebensspanne beträgt etwa beim Hund 12 bis 15 Jahre, beim Pferd rund 30 Jahre und beim Menschen ca. 120 Jahre. Mehrere Alterstheorien versuchen die Fragen zu beantworten, wodurch die maximale Lebensdauer festgelegt wird und welche Prozesse für das Altern verantwortlich sind. Historisch lassen sich diese Theorien zwei Grundkategorien zuordnen: Man unterscheidet einmal Theorien, die Altern als stochastischen Prozess, als „Abnutzungserscheinung“, erklären, wie es z.B. bei der Theorie der freien Radikale und der Mitochondrialtheorie der Fall ist. Dem stehen auf der anderen Seite Theorien gegenüber, die Altern als Ablauf eines genetischen Programms betrachten. In diese Kategorie fallen beispielsweise die Telomerasetheorie oder die Theorie der zellulären Vergreisung (Tab.1). Im Folgenden wird hauptsächlich die Theorie der freien Radikale (Mitochondrialtheorie) ausführlicher erläutert. Detailliert vorgestellt werden die Biologie des Alterns 

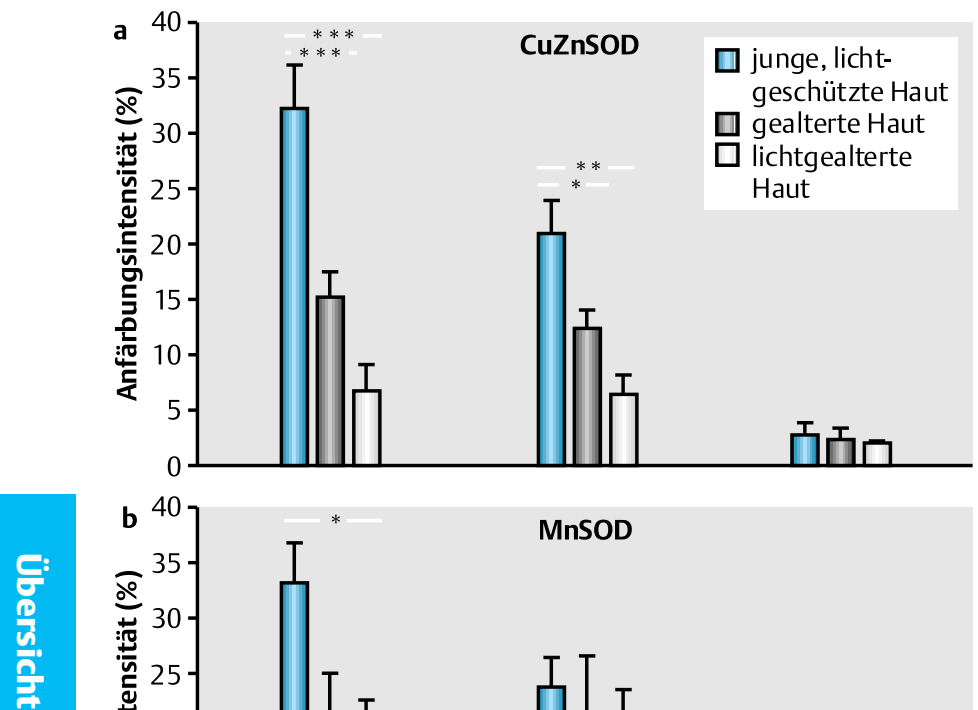

54
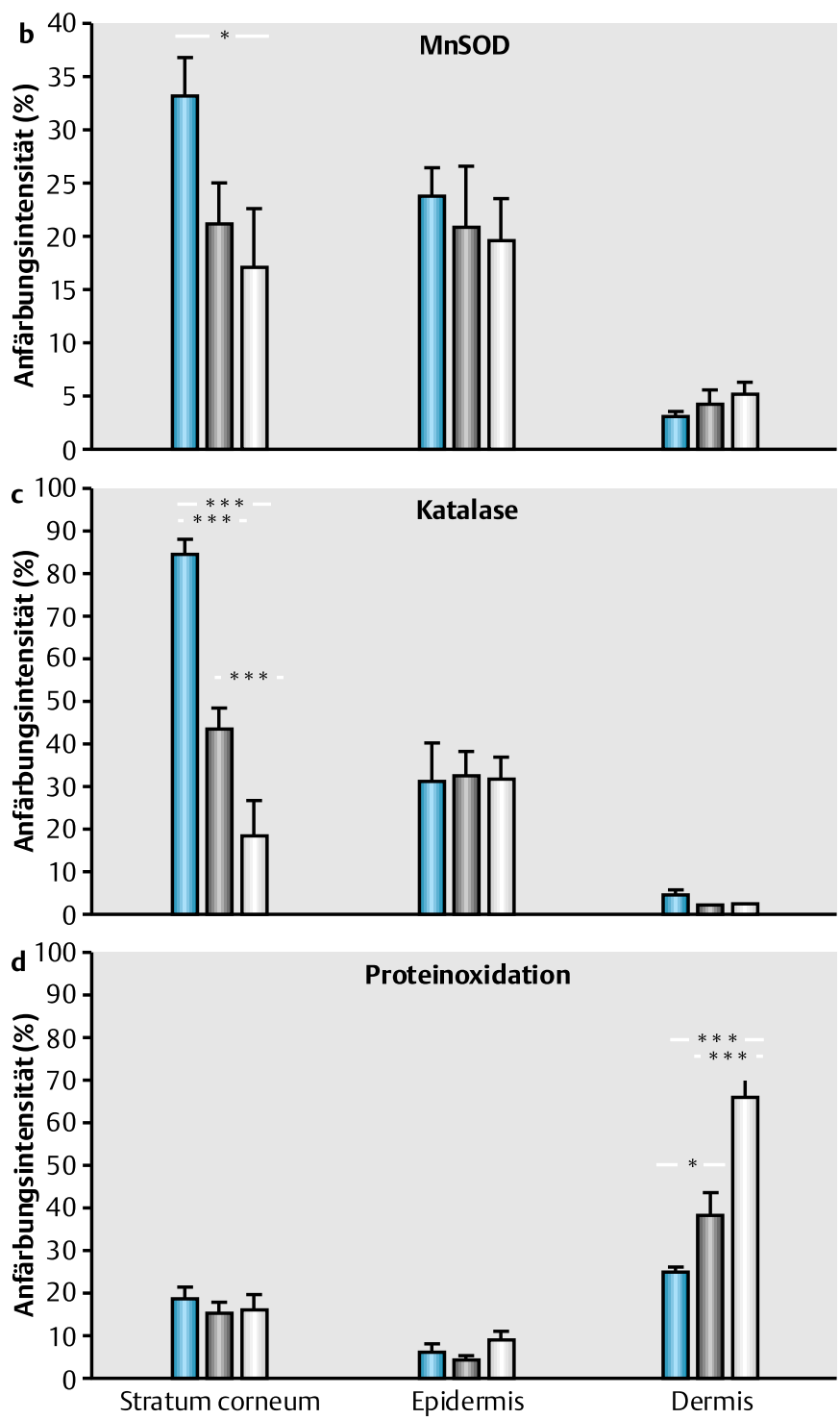

Abb. 2 Alterungsvorgänge in der Haut und daraus resultierende Folgeerscheinungen [2].

und die verschiedenen Alterstheorien von B. R. Troen in einer im letzten Jahr publizierten Übersichtsarbeit [5].

Die vor 50 Jahren von Harmann entwickelte Theorie der freien Radikale basiert auf der Beobachtung, dass im Laufe des Lebens aufgrund der kontinuierlichen Entstehung freier Sauerstoffradikale radikalinduzierte Schäden akkumulieren und letztlich die Körperfunktionen beeinträchtigen [6]. Zwar besitzt der mensch-
Tab. 1 Die verschiedenen Alterstheorien erklären Altern als stochastischen Prozess oder als genetisches Programm

Alterstheorien

Altern als stochastischer Prozess, „Abnutzungserscheinung“

Theorie der freien Radikale

Mitochondrialtheorie

Mutationstheorie

„Error-Catastrophe“

Protein-Modifikation

Altern als genetisches Programm

Hormontheorie/neuroendokrine Theorie

Telomerasetheorie

„genetisch programmierter Selbstmord“/Apoptose

Accelerated Aging Syndroms, Langlebigkeitsgene

Immunologische Theorie

„zelluläre Vergreisung“

liche Organismus zur „Radikalentgiftung“ eine Vielzahl enzymatischer und nicht-enzymatischer Abwehrmechanismen, die in Abb. 3 dargestellt sind. Werden diese jedoch durch oxidativen Stress überlastet, können nicht eliminierte freie Radikale DNASchäden induzieren, die eine protrahierte Alterung auslösen. Nach dieser Theorie ist die Lebenserwartung einer Spezies in erster Linie abhängig von ihrem Grund- und ihrem Sauerstoffumsatz. Dass diese Theorie auch für die Hautalterung relevant ist, erhellt sich aus der Tatsache, dass oxidativer Stress als wichtiger Faktor sowohl bei intrinsischer wie extrinsischer Hautalterung identifiziert wurde.

Der Ort mit der höchsten Generierung an freien Sauerstoffradikalen sind die Mitochondrien, an deren innerer Wand die Energiegewinnung über die Atmungskette stattfindet. Hierbei entsteht permament neben reaktiven Sauerstoffspezies auch $\mathrm{H}_{2} \mathrm{O}_{2}$. Werden diese extrem reaktiven Verbindungen nicht oder erst verzögert eliminiert, so schädigen sie die mitochondriale DNA und damit im Weiteren auch die Funktion der Atmungskette und die Energiegewinnung [7]. Mittlerweile kennt man distinkte Mutationen in der mitochondrialen DNA, die als Langzeitmarker für chronische UV-Schäden und photooxidativen Stress identifiziert wurden. Diese Markermutationen sind vermutlich auch für die verstärkte Expression von Matrixmetalloproteasen von Bedeutung, die den Kollagenabbau verstärken und so zu einer vorzeitigen Erschlaffung und Alterung der Haut führen.

Dagegen erklärt die neuroendokrine Theorie des Alterns (Hormontheorie) den Alterungsprozess als „hormongesteuerte biologische Uhr“ [8]. Bereits ab dem 30. Lebensjahr fallen die Hormonspiegel kontinuierlich ab, was sich in einem Kapazitätsund Funktionsverlust zahlreicher Körpersysteme äußert. Das Hormondefizit betrifft nicht nur die weiblichen Geschlechtshormone (Menopause), sondern auch Androgene (Andropause), Nebennierenrindenhormone (Adrenopause), Wachstumshormone (Somatopause) und Melatonin (Melanopause) [9]. So fällt der Melatonin-Spiegel bereits ab dem 5. Lebensjahr deutlich ab; Wachstumshormone, DHEA (Dehydroepiandrosteron) und IGF 1 (insuline-like growth factor) werden beim Mann etwa ab dem 20. Lebensjahr in immer geringerer Menge produziert. 


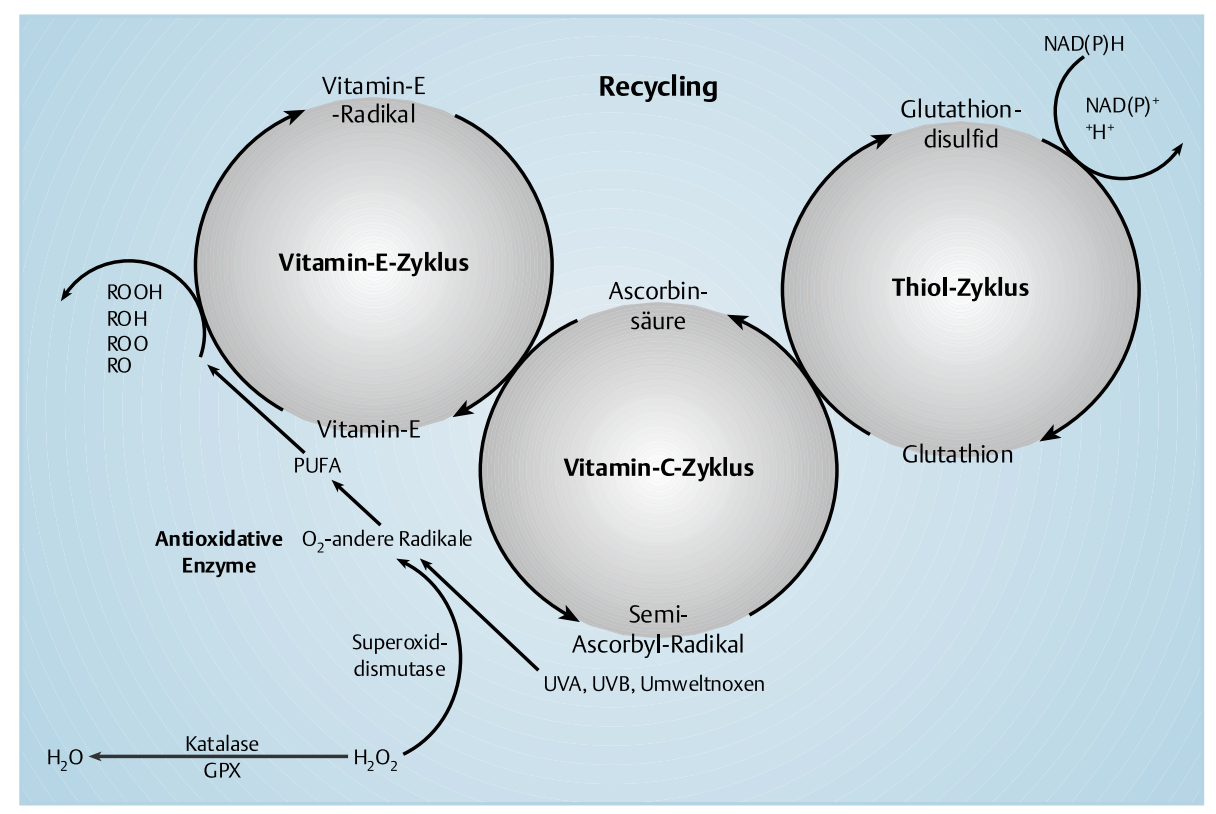

Abb. 3 Das ineinander greifende System enzymatischer und nicht-enzymatischer Radikalfänger im menschlichen Organismus (nach Thiele) [12].

Die Telomerasetheorie betont die wichtige Rolle der Telomere, die die Enden der Chromosomen vor Abbau und Fusion schützen, beim Alterungsprozess [10]. Da sich die Telomeren bei der DNAReplikation kontinuierlich verkürzen und damit „aufbrauchen“, sind Zellteilungen ab einem bestimmten Zeitpunkt nicht mehr möglich. Untergegangene Zellen können somit nicht mehr ersetzt werden. Zwischen dem Alterungsprozess von Zellen und der Telomerlänge konnte mittlerweile ein direkter Zusammenhang etabliert werden. Die wichtige Funktion der Telomeren für das Zellaltern wird durch die Tatsache unterstrichen, dass das Enzym Telomerase, das die Telomerenverkürzung verhindert, in den sich unbegrenzt teilenden Tumorzellen verstärkt exprimiert wird [11].

\section{Literatur}

${ }^{1}$ Statistisches Bundesamt Deutschland 2003.

${ }^{2}$ Hönigsmann H. Einfluss einer Hormonersatz-Therapie auf die Hautalterung. Akt Dermatol 2002; 28: S10-S13

${ }^{3}$ Castelo-Branco $\mathrm{C}$ et al. Relationship between skin collagen and bone changes during aging. Maturitas 1994; 18: 199-206

${ }^{4}$ Imthurn B. Effekte postmenopausaler Hormonveränderungen auf die Haut. Akt Dermatol 2002; 28: S7 -S9

${ }^{5}$ Troen BR. The Biology of Ageing. Mt Sinai J Med 2003; 1: 3-22

${ }^{6}$ Harmann D. Aging: a theory based on free radical and radiation chemistry. J Gerontol 1956; 11: $298-300$

7 Ozawa T. Genetic and functional changes in mitrochondria associated with aging. Physiol Rev 1997; 77: 425-464

${ }^{8}$ Mobbs CV. Neuroendocrinology of aging. In: Schneider EL, Rowe JW, (eds). Handbook of the biology of aging. San Diego (CA): Academic Press, 1996: $234-282$

${ }^{9}$ Phillips TJ, Zeynep D, Malini S. Hormonal Effects on skin aging. Ger Dermatol 17: 661 -673

${ }^{10}$ Ishikawa F. Aging clock: the watchmaker's masterpiece. Cell Mol Life Sci 2000; 57: $698-704$

11 Shay JW. Telomerase in Human Development and Cancer. J Cell Physiol 1997; 173: $266-270$

12 Thiele J. Hautalterung, ein multifaktorielles Phänomen - Antioxidantien. Akt Dermatologie 2002; S1: 3 -9 